\title{
Resistivity due to a Domain Wall in Ferromagnetic Metal
}

\author{
Gen Tatara \\ Graduate School of Science, Osaka University, Toyonaka, Osaka 560, Japan \\ Hidetoshi Fukuyama \\ Department of Physics, University of Tokyo, Hongo, Tokyo 113, Japan
}

(October 2, 2018)

\begin{abstract}
The resistivity due to a domain wall in ferromagnetic metallic wire is calculated based on the linear response theory. The interaction between conduction electrons and the wall is expressed in terms of a classical gauge field which is introduced by the local gauge transformation in the electron spin space. It is shown that the wall contributes to the decoherence of electrons and that this quantum correction can dominate over the Boltzmann resisitivity, leading to a decrease of resisitivity by nucleation of a wall. The conductance fluctuation due to the motion of the wall is also investigated. The results are compared with recent experiments.
\end{abstract}

72.10.Fk, 75.60.Ch, 72.15.Rn, 75.45.+j

Typeset using REVTEX 
The interplay between the electron transport properties and the magnetic object such as a magnetization and a domain wall(DW) has recently been attracting much attention. Of particular interest is the case of mesoscopic system where the motion of a DW or a magnetization can be driven by quantum fluctuation and described as a macroscopic quantum phenomena [1]. In the case of the quantum depinning of a DW [2], for example, a theoretical study [3] indicates that the depinning can be affected by the dissipation caused by the conduction electron if the thickness of the wall, $\lambda$, is small, e.g., a few lattice constants. The change of the magnetization associated with such a depinning of a mesoscopic DW is very small and so it is very difficult to observe such small magnetic objects directly, e. g. by SQUID. The transport properties on the other hand can detect a very small change of the magnetization as a change of resistance. Indeed recently in a mesoscopic wire of $\mathrm{Ni}$ with diameter of $300 \AA$ several small discontinuous changes of the resistivity have been observed as the magnetic field is swept [4]. It is argued there that these jumps are due to the change of the total magnetization by depinning of a DW, and the displacement of the wall has been estimated from the value of magnetoresistance to be $\sim 1.2 \mu \mathrm{m}$ [4]. Other possible origins of this jump are proposed in this paper.

Not only in these mesoscopic system the interplay between the magnetic structure and the electronic transport properties may play important roles in the bulk system; e. g., in double exchange systems like $\mathrm{La}_{1-x} \mathrm{Sr}_{x} \mathrm{MnO}_{3}$, scattering by DWs is considered as a possible origin of low temperature magnetoresistance [5].

In this paper we study the resistivity in ferromagnetic metals arising from the scattering by a DW on the basis of linear response theory by taking account of the impurity scattering at the same time. The case of $\lambda \ll l$ ( $l$ being the elastic mean free path) has been studied by Cabrera and Falicov [6] in the classical Boltzmann approximation. Their result indicates that the resisitivity becomes large only when the spin splitting is comparable to the Fermi energy and $k_{F} \lambda \lesssim 1\left(k_{F}\right.$ being the Fermi momentum). In thier study, however, electronic motions have been assumed to be one-dimensional, which is not realistic, at least at present, in actual metallic wires. Here we study the effect of a DW on resistivity in a mesoscopic wire 
with width $L_{\perp}$ satisfying $\lambda \gtrsim L_{\perp} \gg k_{F}^{-1}$, thus treating the electron as three-dimensional. The length is $L$ and the wire direction has been chosen as $z$-axis. We investigate the quantum corrections to the resistivity by a wall as well as the Boltzmann resistivity. The conductance fluctuations [7,8] arising from the motion of the wall has also been calculated.

We consider explicitly the case described by a single-band Hubbard model in the HartreeFock approximation [9]. The calculation is carried out at zero temperature. The Lagrangian of the electron (denoted by $c^{(0)}$ ) in the imaginary time is given as

$$
L=\sum_{\mathbf{k} \sigma} c_{\mathbf{k} \sigma}^{(0) \dagger}\left(\partial_{\tau}+\epsilon_{\mathbf{k}}\right) c_{\mathbf{k} \sigma}^{(0)}-U \sum_{\mathbf{x}} \boldsymbol{M}\left(c^{(0) \dagger} \boldsymbol{\sigma} c^{(0)}\right)
$$

where $\epsilon_{\mathbf{k}} \equiv \hbar^{2} \mathbf{k}^{2} / 2 m-\epsilon_{F}\left(\epsilon_{F}\right.$ being the Fermi energy) and $U$ is the Coulomb interaction. The spin index is denoted by $\sigma= \pm$ and $\boldsymbol{\sigma}$ is the Pauli matrix. The magnetization vector, $\boldsymbol{M}$, is in unit of Bohr magneton. The configuration of $\boldsymbol{M}$ is determined by the ferromagnetic Heisenberg model [3],

$$
H_{M}=\sum_{\mathbf{x}}\left[\frac{J}{2}|\nabla M|^{2}-\frac{K}{2} M_{z}^{2}\right]
$$

where $J$ is the effective exchange energy determined by $U$ and $K$ is the magnetic anisotropy energy introduced phenomenologically [10]. Here we are interested in the solution of a single domain wall. In terms of the polar coordinate that represents the direction of magnetization vector, $(\theta, \phi)$, the solution of a DW is given by $\cos \theta=\tanh \frac{z}{\lambda}$ and constant $\phi$, where $\lambda=\sqrt{K / J}$.

In Eq. (1) the last term represents the interaction between the magnetization and the electron. For the perturbative calculation of resistivity, we need to rewrite this term by use of the local gauge transformation in the spin space,

$$
c_{\sigma} \equiv \sigma\left(\cos \frac{\theta}{2} c_{\sigma}^{(0)}-i \sin \frac{\theta}{2} c_{-\sigma}^{(0)}\right) .
$$

In terms of the new electron operator, $c$, the Lagrangian is written as $[3] L=\sum_{\mathbf{k} \sigma} c_{\mathbf{k} \sigma}^{\dagger}\left(\partial_{\tau}+\right.$ $\left.\epsilon_{\mathbf{k} \sigma}\right) c_{\mathbf{k} \sigma}+H_{\text {int }}$, where $\epsilon_{\mathbf{k} \sigma} \equiv \epsilon_{\mathbf{k}}-\sigma \Delta$ with $\Delta \equiv U|\boldsymbol{M}|$ being half the splitting between the up and down spin electrons. The interaction is obtained as 


$$
H_{\mathrm{int}}=\frac{\hbar^{2}}{2 m} \sum_{\mathbf{k}} \sum_{q / / z}\left(-\left(k_{z}+\frac{q}{2}\right) a_{q} c_{\mathbf{k}+q}^{\dagger} \sigma_{x} c_{\mathbf{k}}+\frac{1}{4} \sum_{p / / z} a_{p} a_{-p+q} c_{\mathbf{k}+q}^{\dagger} c_{\mathbf{k}}\right) .
$$

Here $a_{q} \equiv(1 / V) \sum_{\mathbf{x}} e^{-i q z} \nabla_{z} \theta=(\pi / L) e^{-i q z_{i}}[1 / \operatorname{ch}(\pi q \lambda / 2)]\left(V \equiv L_{\perp}^{2} L\right.$ and $z_{i}$ being the center coodinate of the domain wall). Due to this gauge transformation, the electronic current in $z$-direction is changed to be $J_{z}=J_{z}^{0}+\delta J$, where $J_{z}^{0} \equiv(e \hbar / m) \sum_{\mathbf{k}} k_{z} c_{\mathbf{k}}^{\dagger} c_{\mathbf{k}}$ and

$$
\delta J \equiv-\frac{e \hbar}{2 m} \sum_{\mathbf{k}, q / / z} a_{q} c_{\mathbf{k}+q}^{\dagger} \sigma_{x} c_{\mathbf{k}} .
$$

By use of the Kubo formula, the conductivity for the current along the wire is calculated from the current-current correlation function. By assuming that the scattering due to normal impurities are dominant, we estimate the effect of DW on the correction to the conductivity perturbatively to the second order of $a$. (The first order contribution vanishes.) The second order contributions to the Boltzmann conductivity are shown in Fig. 11. The process $Q_{1}$ arises from the correlation of $\delta J$ and $Q_{3}$ is due to $\delta J$ and an interaction with the wall. $Q_{2}$ and $Q_{4}$ are the self-energy corrections due to the wall and $Q_{5}$ is the vertex correction to the correlation of $J_{z}^{0}$. After straightforward calculation and by use of the particle-hole symmetry, which we assume, $\Delta Q \equiv \sum_{i=1}^{5} Q_{i}$ is shown to be

$$
\Delta Q\left(i \omega_{\ell}\right)=\frac{1}{2}\left(\frac{e \hbar \Delta}{m}\right)^{2} \frac{1}{\beta} \sum_{n} \frac{1}{V} \sum_{\mathbf{k} q \sigma}\left|a_{q}\right|^{2} G_{\mathbf{k}-\frac{q}{2}, n, \sigma} G_{\mathbf{k}-\frac{q}{2}, n+\ell, \sigma} G_{\mathbf{k}+\frac{q}{2}, n,-\sigma} G_{\mathbf{k}+\frac{q}{2}, n+\ell,-\sigma} .
$$

Here $\omega_{\ell} \equiv 2 \pi \ell / \beta$ and the Green function is given by $G_{\mathbf{k}, n, \sigma} \equiv 1 /\left[i\left(\varepsilon_{n}+(\hbar / 2 \tau) \operatorname{sgn}(n)\right)-\right.$ $\left.\epsilon_{\mathbf{k k k k} \sigma}\right]$, where $\varepsilon_{n}=\pi(2 n+1) / \beta$ and $\tau$ is the life time due to the normal impurity scattering and $\operatorname{sgn}(n)=1$ and -1 for $n>0$ and $n<0$, respectively.

Hence the correction to the Boltzmann conductivity by a DW, $\Delta \sigma$, is obtained as $\Delta \sigma=$ $-\sigma_{0} A$ where $\sigma_{0} \equiv e^{2} n \tau / m$ ( $n$ being the electron density) is the Boltzmann conductivity without the wall and $A$ is given by

$$
A=\frac{\pi}{\hbar} \frac{\Delta^{2} \tau}{2 n V} n_{\mathrm{w}} \sum_{\sigma, \pm} \frac{N_{\sigma}}{k_{\mathrm{F} \sigma}} \int_{0}^{\infty} \frac{d x}{x} \frac{1}{\operatorname{ch}^{2} x} \tan ^{-1}\left(\frac{2 l_{\sigma}}{\pi \lambda} x \pm 2 \Delta \frac{\tau}{\hbar}\right) .
$$

Here $l_{\sigma} \equiv \hbar k_{F \sigma} \tau / m, n_{\mathrm{w}} \equiv 1 / L$ being the density of the wall and $N_{\sigma} \equiv\left(m k_{F \sigma} V / 2 \pi^{2} \hbar^{2}\right)$ is the density of states at the Fermi energy of the electron with spin $\sigma$. The wall contribution to the resisitivity is given as $\rho_{\mathrm{w}} \equiv \sigma_{0}^{-1}\left((1-A)^{-1}-1\right) \simeq \sigma_{0}^{-1} A$. 
We consider a ferromagnet where $\Delta \tau / \hbar \gg 1$ is satisfied. Then Eq. (7) reduces to

$$
A \simeq \frac{3 n_{\mathrm{w}}}{2 m n \lambda} \sum_{\sigma} \frac{N_{\sigma}}{V}
$$

Let us look into the effect of the wall on qunatum transport properties in disordered system, where the interference effect, which is represented by the maximally-crossed diagram (Cooperon), becomes important. The processes which describe the effect of the wall on the qunatum correction at low energy are shown in Fig. 2. They both contribute to the dephasing of the electron, but the vertex type process (b) includes Cooperons which connect the electrons with differrent spin, and thus is suppressed in ferromagnets we are considering due to the condition $\Delta \tau / \hbar \gg 1$. Hence only the self-energy type (a) is important here. The higher order contributions similar to this process can be summed up giving rise to the mass of the Cooperon. The quantum correction by the wall is then obtained as

$$
\sigma_{Q}=\frac{2 \hbar e^{2} k_{F}^{2} \tau}{3 \pi m^{2}} \frac{1}{V} \sum_{q}\left(\frac{1}{D q^{2}}-\frac{1}{D q^{2}+\frac{1}{\tau_{\mathrm{w}}}}\right)
$$

where $D \equiv k_{F}^{2} \tau / 3$ and $\tau_{\mathrm{w}}$ is the life-time due to the wall given by $1 / \tau_{\mathrm{w}}=\left(n_{\mathrm{w}} k_{F}^{2} \hbar^{4} / 6 \Delta^{2} \lambda \tau\right)$. In the case where $D L_{\perp}^{-2}>\tau_{\mathrm{w}}^{-1}$, which we assume, the $q$-summation should be carried out along the one-dimention with a cut off of $L^{-1}$ for small $q$. The result for $L / l \gg 1$ and $\kappa \equiv \tau / \tau_{\mathrm{w}} \ll 1$ is

$$
\frac{\sigma_{Q}}{\sigma_{0}} \simeq \frac{6}{k_{F}^{2} L_{\perp}^{2}}\left(\frac{L}{l}-\frac{\tan ^{-1}(\sqrt{3 \kappa} L / l)}{\sqrt{3 \kappa}}\right) .
$$

Note that $\sigma_{Q}$ is positive, sunce the DW suppresses the interference due to random impurity scattering.

So far we have studied a static wall. Let us now discuss the conductance fluctuation due to the motion of the wall. In this case a small jump of a wall can result in substantial change in resistivity, in contrast to the change due to the effect of classical magnetoresisitance [4], which becomes important only when the wall moves over a distance comparabel to $L$. The calculation goes in the siminlar way as the conductance change due to the motion of a single atom in a disordered metal $[8]$. The square of the conductance change $\delta G$ due to 
the motion of a wall over a distance of $r$ is evaluated by calculating the diagram with two bubbles with the wall position at $z=r$ and $z=0$ connected by impurities and the wall. A typical diagram is shown in Fig. 3. The DW line here represents the motion of the wall and Cooperons include the mass arising from the wall, $1 / \tau_{\mathrm{w}}$. There are other diagrams with the contribution of the same order which contains one or two more impurity ladders [7] and the result of $\delta G$ is obtained as

$$
\frac{\delta G(r)}{e^{2} / h}=\sqrt{2} \frac{4 \pi}{3} \epsilon^{2} \kappa \alpha \times\left\{\begin{array}{cc}
\frac{1}{6}\left(\frac{r}{\lambda}\right)^{2} & (r \ll l, \lambda) \\
1 & (r \gg l, \lambda)
\end{array},\right.
$$

where $\epsilon \equiv l / L$ and $\alpha \equiv\left[\sum_{q}\left(D q^{2} \tau+\kappa\right)^{-4}\right]^{(1 / 2)}$ is calculated for $\epsilon, \kappa \ll 1$ as

$$
\alpha \simeq \frac{9}{2 \pi} \frac{1}{\epsilon \kappa}\left(\frac{5}{24 \kappa^{2}}\left(\frac{\tan ^{-1}(\epsilon / \sqrt{3 \kappa})}{\sqrt{3 \kappa}}-\frac{\epsilon\left(\epsilon^{2}+5 \kappa\right)}{\left(\epsilon^{2}+3 \kappa\right)^{2}}\right)-\frac{\epsilon}{\left(\epsilon^{2}+3 \kappa\right)^{3}}\right) .
$$

Let us give an numerical estimate of our theoretical conclusions. Consider a wire of $\mathrm{Ni}$ or Fe with $L=10 \mu \mathrm{m}$ and $L_{\perp}=300 \AA$, where $\lambda \sim 500 \AA$ [4]. If we consider $d$ electron $\left(k_{F}^{-1} \sim 1.5 \AA, \Delta / \epsilon_{F} \sim 0.2\right)$ and choose $l \sim 1000 \AA$, then $\Delta \tau=150$ and Eq. (8) leads to a very small Boltzmann contribution of $A \simeq 1.4 \times 10^{-8}$. For $s$ electron, $\Delta / \epsilon_{F}$ will be smaller by a factor of about $10^{-2}$ and then from Eq. (7) we obtain $A \simeq 2.7 \times 10^{-9}$. On the other hand, the quantum correction becomes larger for the above values of parameters $\left(\kappa\right.$ is $\left.\sim 3.7 \times 10^{-4}\right)$; $\sigma_{Q} / \sigma_{0}=1.6 \times 10^{-3}$. Thus DWs will contribute to a decrease of resisitivity in a ferromagnetic wire of transition metals. If the wall moves over a distance of $r \sim 100 \AA$ in this situation, the expected conductance change is $\delta G \simeq 5.0 \times 10^{-3}\left(e^{2} / h\right)$.

In the experiment on $\mathrm{Ni}$ [4], a descrete increase of resisitivity of about $0.2 \%(\delta \rho \simeq$ $2 \times 10^{-9} \Omega \mathrm{cm}$ or $\left.\delta G \simeq 5 \times 10^{-3}\left(e^{2} / h\right)\right)$ has been observed as the magnetic field is swept above the coercive field, at which the minimum of resistivity appears. Comparison with our study may suggest two possibilities for the cause. One is that $\delta \rho$ might be due to the annihilation of a wall. The other is that $\delta \rho$ can be the fluctuation due to a motion of wall over a distance of $r \sim 100 \AA$. Further studies are needed to determine which is the true origin. In this context it is interesting to note that a recent experiment on Fe wire with width of $3000 \AA$ has disclosed the existence of a negative jump of $\rho$ followed by a positive one 
close to the field where $\rho$ becomes minimum [11]. This result may suggest that the jumps are due to the nucleation and subsequent annihilation of a wall.

Our study indicates that not only in mesoscopic case the magnetoresisitance of bulk ferromagnets can be affected by the dephasing effect by DWs in particular close to the coercive field, where $M=0$ and thus the system will contain many domains.

To summarize, the resistivity arising from the scattering of the conduction electron by a domain wall in a wire of ferromagnetic metal is calculated based on the linear response theory. The interaction with the wall is expressed as a classical gauge field acting on the electron, which we examined in the second order perturbation theory. In addition to the Boltzmann resisitivity, we have investigated the effect of the wall on quantum transport properties in disordered metals. The wall suppresses the intereference between the electron, and hence decreases the resisitivity in the weakly localized regime. It will be interesting to observe in magnetic wires this reduction of resistivity by the nucleation of domains in more definitive ways. It has been shown that small motion of a wall can lead to substantial conductance fluctuation. The present calculation provides a first quantitative estimate of the effect of a domain wall on the mesoscopic transport properties, which, we hope, will be useful in the interpretation of the experimental results in the near future.

Authors thank K. Kuboki, H. Kohno, Y. Otani and K. Takanashi for valuable discussions. G. T. thanks The Murata Science Foundation for finantial support. 


\section{REFERENCES}

[1] See Quantum Tunneling of Magnetization, L. Gunther and B. Barbara (eds.) (Proc. NATO workshop, Chichilianne, France, June 1994), Kluwer Publishing (1995).

[2] P. C. E. Stamp: Phys. Rev. Lett. 66, 2802 (1991).

[3] G. Tatara and H. Fukuyama: Phys. Rev. Lett. 72, 772 (1994); J. Phys. Soc. Jpn. 63, 2538 (1994).

[4] N. Giordano and J. D. Monnier, Physica B194-196, 1009 (1994); K. Hong and N. Giordano, Phys. Rev. B51, 9855 (1995); J. Phys. CM8, 301 (1996).

[5] P. Schiffer et al., Phys. Rev. Lett. 753336 (1995).

[6] G. G. Cabrera and L. M. Falicov, Phys. Stat. Sol. (b)61, 539 (1974).

[7] P. A. Lee and A. D. Stone, Phys. Rev. Lett. 55, 1622 (1985); P. A. Lee, A. D. Stone and H. Fukuyama, Phys. Rev. B35, 1039 (1987).

[8] S. Feng, P. A. Lee and A. D. Stone, Phys. Rev. Lett. 56, 1960 (1986).

[9] In the case with multi-bands like $s$ - $d$ model, the results turns out to be valid if $U$ in the Hubbard model is replaced by the $s$ - $d$ coupling.

[10] The choice of $z$-axis as an easy axis is due to the demagnetization field in the case of the wire geometry. The transverse anisotropy energy is neglected here, since it does not affect our calculation of a static wall.

[11] Y. Otani, private communications. 


\section{FIGURES}

FIG. 1. The contributions to the Boltzmann conductivity which are the second order with respect to the interaction with the domain wall, denoted by wavy lines. Solid lines indicate the electron Green functions and the current vertex (expressed by crosses) with wavy line represents $\delta J$.

FIG. 2. (a): The dominant process to the qunatum correction of the conductivity. Hatched square denotes the particle-particle ladder (Cooperon) due to the impurity scattering. Process (b), which contains Cooperons connecting the electrons with different spin (denoted by $\sigma$ and $-\sigma$ ), is unimportant in ferromagnets due to the present condition $\Delta \tau / \hbar \gg 1$.

FIG. 3. An example of diagrams which contributes to the conductance fluctuation due to the motion of the wall. Wavy lines represent the motion of the domain wall and the Cooperons here include the mass due to the domain wall. 


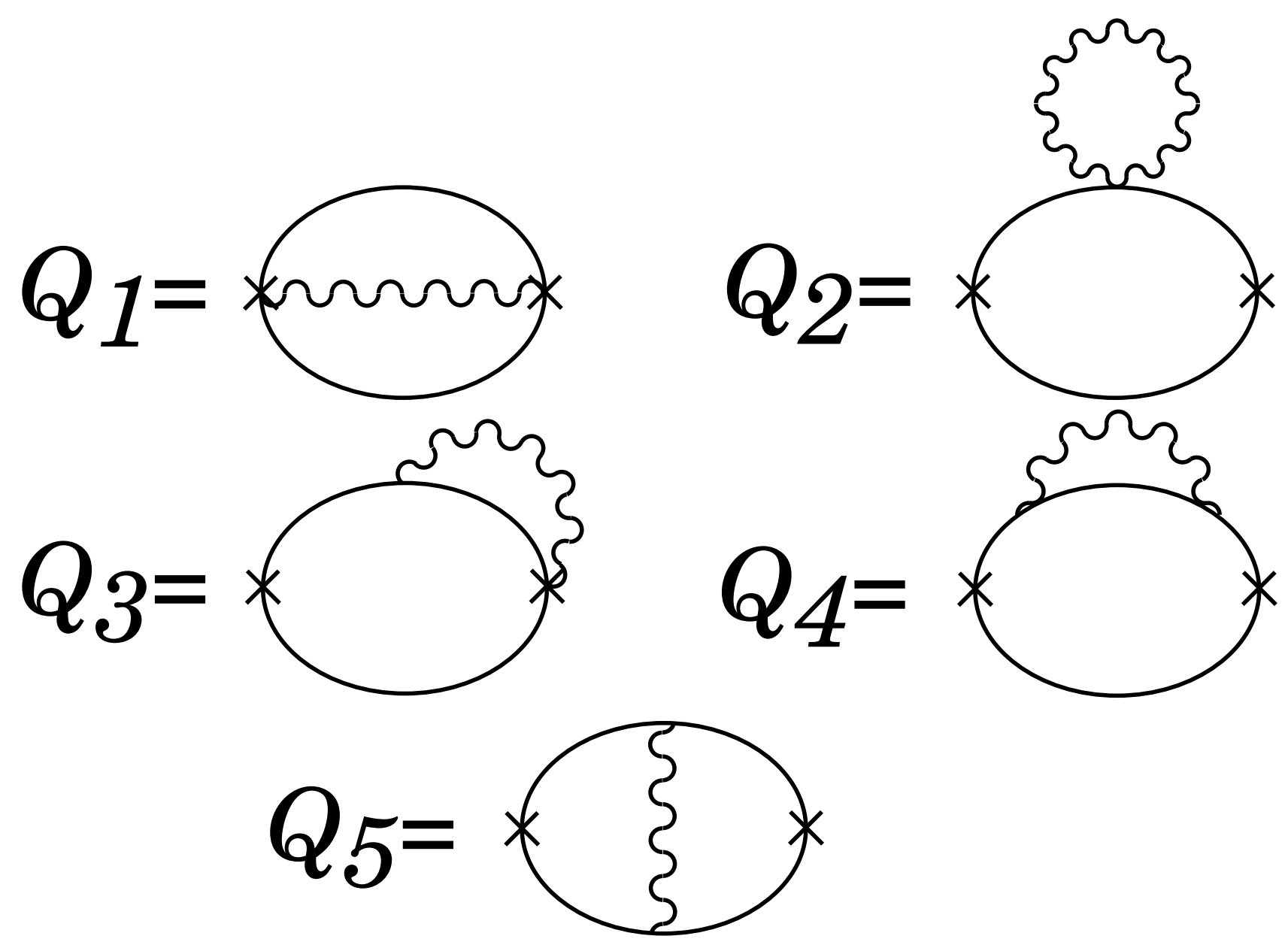

Tatara and Fukuyama Fig. 1 


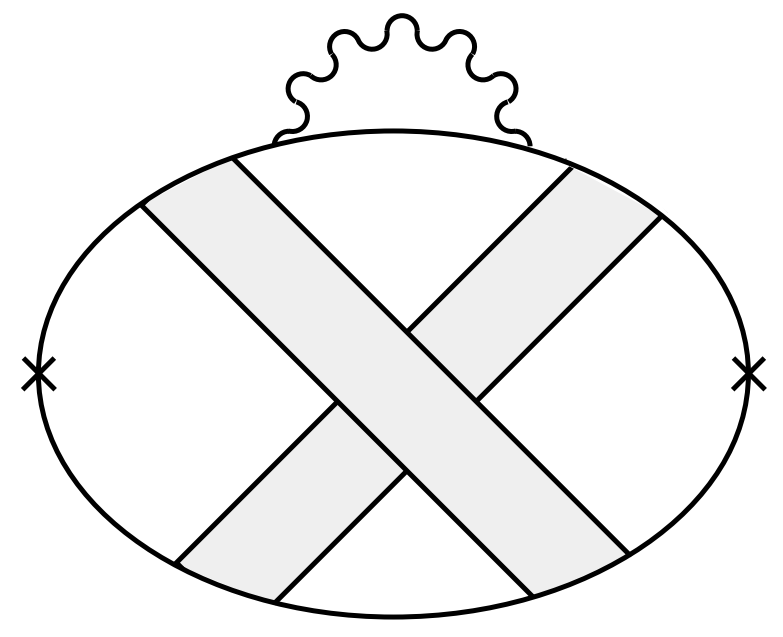

(a)

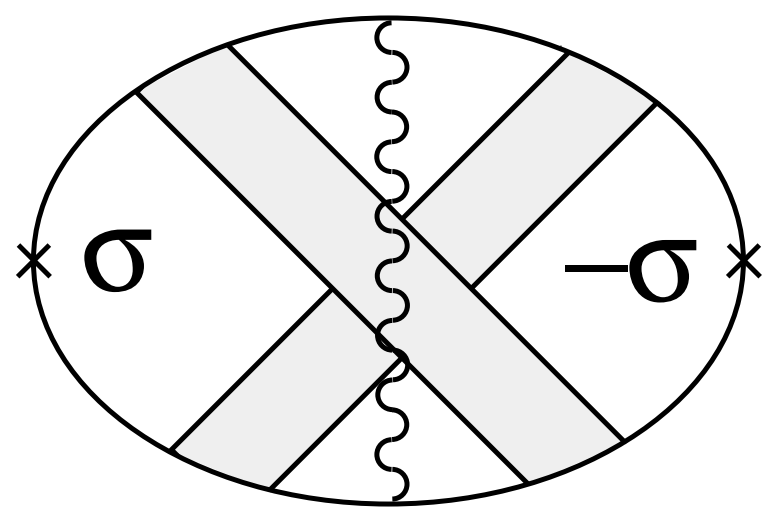

(b)

Tatara and Fukuyama Fig. 2 


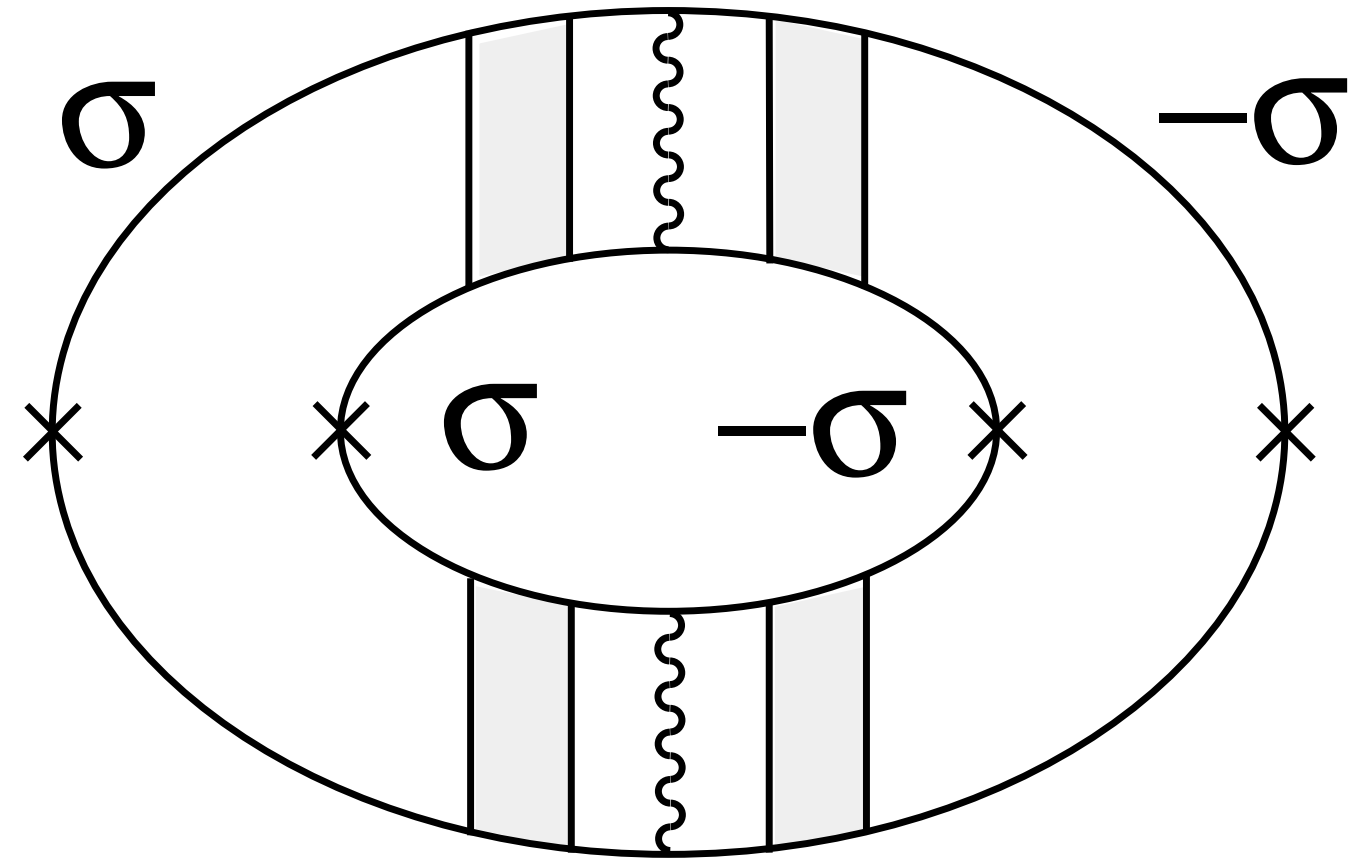

Tatara and Fukuyama Fig. 3 